\title{
Semiconductor Lasers With Integrated Plasmonic Polarizers
}

\section{Citation}

Yu, Nanfang, Qi Jie Wang, Christian Pflügl, Laurent Diehl, Federico Capasso, Tadataka Edamura, Shinichi Furuta, Masamichi Yamanishi, Hirofumi Kan. 2009. Semiconductor lasers with integrated plasmonic polarizers. Applied Physics Letters 94: 151101.

\section{Published Version}

doi:10.1063/1.3093476

\section{Permanent link}

http://nrs.harvard.edu/urn-3:HUL.InstRepos:3445982

\section{Terms of Use}

This article was downloaded from Harvard University's DASH repository, and is made available under the terms and conditions applicable to Other Posted Material, as set forth at http:// nrs.harvard.edu/urn-3:HUL.InstRepos:dash.current.terms-of-use\#LAA

\section{Share Your Story}

The Harvard community has made this article openly available.

Please share how this access benefits you. Submit a story.

Accessibility 


\title{
Semiconductor lasers with integrated plasmonic polarizers
}

\author{
Nanfang Yu, ${ }^{1, a)}$ Qi Jie Wang, ${ }^{1}$ Christian Pflügl, ${ }^{1}$ Laurent Diehl, ${ }^{1}$ Federico Capasso, ${ }^{1, b)}$ \\ Tadataka Edamura, ${ }^{2}$ Shinichi Furuta, ${ }^{2}$ Masamichi Yamanishi, ${ }^{2}$ and Hirofumi Kan ${ }^{2}$ \\ ${ }^{1}$ School of Engineering and Applied Sciences, Harvard University, Cambridge, Massachusetts 02138, USA \\ ${ }^{2}$ Central Research Laboratory, Hamamatsu Photonics K.K., Hamamatsu 434-8601, Japan
}

(Received 13 November 2008; accepted 11 February 2009; published online 13 April 2009)

\begin{abstract}
The authors reported the plasmonic control of semiconductor laser polarization by means of metallic gratings and subwavelength apertures patterned on the laser emission facet. An integrated plasmonic polarizer can project the polarization of a semiconductor laser onto other directions. By designing a facet with two orthogonal grating-aperture structures, a polarization state consisting of a superposition of a linearly and right-circularly polarized light was demonstrated in a quantum cascade laser; a first step toward a circularly polarized laser. (C) 2009 American Institute of Physics.

[DOI: $10.1063 / 1.3093476$ ]
\end{abstract}

Light sources with a desirable polarization state are of high interests for many applications. For example, satellite communications use dual polarizations to double the capacity of the services, ${ }^{1}$ circularly polarized light sources are of great importance in chemistry and biology for detecting molecules exhibiting circular dichroism, ${ }^{2}$ and laser sources with a variety of polarization states are used for quantum cryptography. ${ }^{3}$ However, semiconductor lasers are mostly linearly polarized either TE or TM, which is determined by the optical selection rules of the gain medium. ${ }^{4,5}$ Other states of polarizations proved difficult to achieve.

Conventionally, manipulation of the polarization state of a light output is conducted externally using bulky and expensive optical components such as beam-splitting polarizers and wave plates. Previously, elliptical aperture arrays defined in a metal film have been shown to serve as a linear polarizer. ${ }^{6,7}$ A single aperture surrounded by elliptical corrugations has been demonstrated to function as a quarter wave plate. $^{8}$ The integrated plasmonic polarizer demonstrated in the present work provides a compact solution allowing the development of polarization-controllable active and passive devices over a wide frequency range, from the visible to the far infrared (IR). The design involves the integration of an aperture-grating plasmonic structure on the emission surface of a light source.

We used mid-IR edge-emitting quantum cascade lasers (QCLs) with two objectives in mind: First is to filter the linearly polarized output of a QCL to produce linearly polarized emission along other directions and second to transform a linearly polarized QCL into a circularly polarized device. QCLs are based on intersubband transitions between quantized electronic levels confined in the conduction band of a semiconductor heterostructure. As such, QCLs are intrinsically TM polarized. The electric field of the mode is parallel to the growth direction of the heterostructure $(z$ direction in Fig. 1)..$^{5}$ To project the polarization of a QCL onto a direction defined by $\theta$, an aperture-grating plasmonic structure ${ }^{9}$ similar to that reported in Ref. 10 is used except that in the present work the normal to the slit aperture and the grating grooves

\footnotetext{
${ }^{a}$ Electronic mail: nyu @fas.harvard.edu

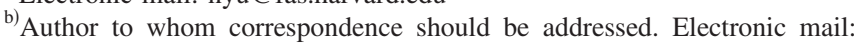
capasso@ seas.harvard.edu.
}

is at an angle $\theta$ from the vertical direction, as shown in Fig. 1. The aperture is defined on the laser active region and is subwavelength in one dimension and comparable to the free space wavelength in the other dimension (Fig. 1). Only the component of the laser polarization perpendicular to the grooves couples to surface plasmons (SPs) propagating along the grating. The output of the device is thus an interference pattern produced by direct emission from the aperture and re-emissions from the grating grooves due to Bragg scattering of SPs by the second-order grating. The polarization of the re-emitted light is along the $\theta$ direction, determined by the orientation of the grooves. The polarization of the direct emission from the aperture is mainly polarized in the direction given by $\theta$ because the power throughput of the field component perpendicular to the aperture slit is significantly larger than that of the field component parallel to it. ${ }^{11}$ As a result, the output of the device is polarized along the $\theta$ direction. It should be noted that an aperture without the grating would result in radiation mainly polarized in the $\theta$ direction but with large beam divergence. The grating greatly reduces the beam divergence angle along the $\theta$ direction due to the interference effect. ${ }^{10,12,13}$

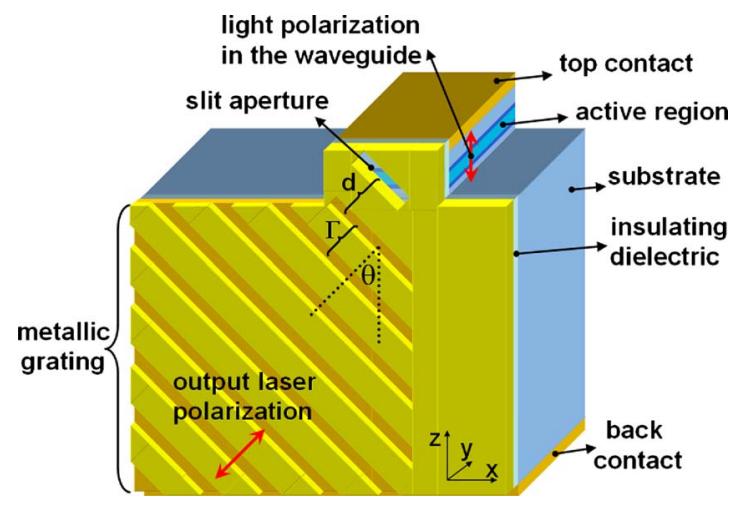

FIG. 1. (Color online) A schematic of a plasmonic polarizer integrated onto the facet of a QCL. The linear polarization direction of the output light is determined by the orientation of the slit aperture and the grating $\theta . d$ is the distance between the aperture and the first grating groove and $\Gamma$ is the grating period. The optimal parameters for $\lambda=9.9 \mu \mathrm{m}$ QCLs are $d$ $=7.1 \mu \mathrm{m}, \Gamma=9.4 \mu \mathrm{m}$, groove depth $h=1.5 \mu \mathrm{m}$, groove width $w$ $=0.8 \mu \mathrm{m}$, metal film thickness $600 \mathrm{~nm}$, insulating dielectric film (alumina) thickness $200 \mathrm{~nm}$. 


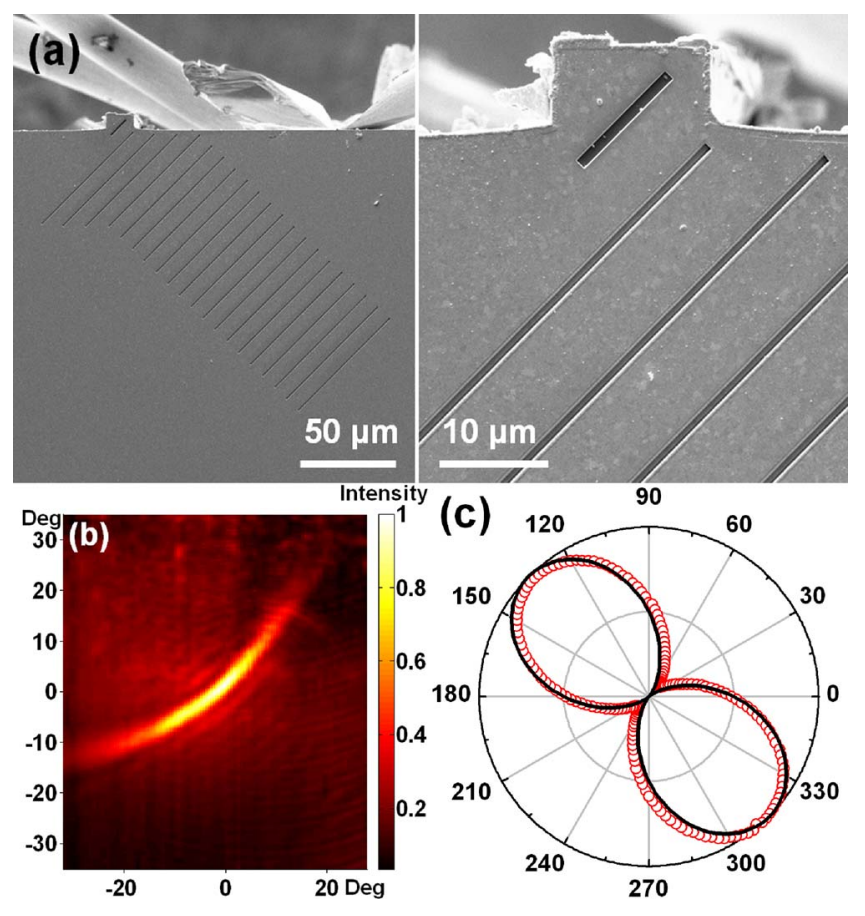

FIG. 2. (Color online) (a) Electron micrographs of the facet of a QCL integrated with a plasmonic polarizer. The orientation of the slit aperture and the grating is $45^{\circ}$ with respect to the vertical direction. Right panel is the zoom-in view of the left panel. The aperture has a size of $1.1 \times 11.5 \mu \mathrm{m}^{2}$. (b) A 2D far-field emission pattern of the device. (c) Measured device output power as a function of the rotation angle of the wire-grid polarizer $\alpha(\alpha$ $=0^{\circ}$ corresponds to the $+x$ direction). Open circles are experimental data and solid curve is calculation for a $45^{\circ}$ linearly polarized light.

Several geometric parameters need to be optimized to achieve the highest power throughput, including the distance between the aperture and the nearest groove $d$, the grating period $\Gamma$, the width $w$, and the depth $h$ of the grooves. The physical considerations used to determine these parameters were discussed in details in Refs. 10 and 12. A list of the optimal values is given in the caption of Fig. 1. To fabricate the device, we used a method described in Refs. 10, 12, and 13. The grating grooves were cut on the bare device facet using focused ion beam (FIB) milling, then an insulating dielectric layer and a gold film were deposited, and finally FIB was used again to open the aperture. The QCLs used operate on a single transverse mode at $\lambda=9.9 \mu \mathrm{m}$.

Figure 2(a) shows electron micrographs of the facet of a QCL patterned with a plasmonic polarizer with $\theta=45^{\circ}$. Figure 2(b) is the measured two-dimensional (2D) far-field emission profile of the device. It is observed that the beam divergence is reduced along the $\theta=45^{\circ}$ direction. In order to determine the polarization state of the beam, a mid-IR detector with a wire-grid polarizer placed in front of it was positioned in the far field where the laser intensity is maximum. As the axis of the wire-grid polarizer was rotated, the signal on the detector showed two clear maxima and minima as summarized in Fig. 2(c). The maxima occurred at a rotation angle corresponding to $\alpha=135^{\circ}$ and $-45^{\circ}$ with respect to the $+x$ direction, and we observed a high cross-polarization suppression ratio, demonstrating our ability to efficiently project the polarization of a QCL onto other directions. The maximum power output of the device is about $30 \%$ of that of the original laser due to reduced overlap between the tilted aperture and the active region of the laser as compared to the

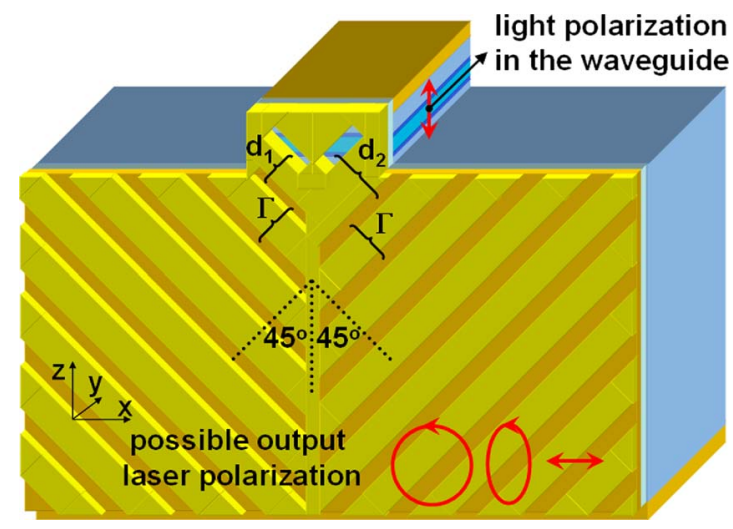

FIG. 3. (Color online) A schematic of a QCL integrated with a plasmonic polarizer capable of producing circularly, elliptically, or TE polarized light. $d_{1}$ and $d_{2}$ are distances from the apertures to the first grating grooves on the left and right, respectively, and $\Gamma$ is the grating period. The polarization state of the output light can be controlled by tuning $d_{1}$ and $d_{2}$.

case of an aperture parallel to the active region as in the case of one-dimensional collimation. ${ }^{10}$ The power of the main lobe of the far field (the crescent) contains approximately $30 \%$ of the total emitted power. The laser threshold of the device is essentially the same as the original laser. Note that the fraction of the laser power coupled into SPs through the aperture decreases with increasing $\theta$, thus the design cannot work properly to produce TE polarized light. Instead one may use a design based on a structure consisting of two apertures and gratings, see the discussion later.

A circularly polarized beam can be constructed by coherently combining two linearly polarized beams satisfying three conditions. The two beams should have perpendicular polarizations, $90^{\circ}$ phase difference, and the same amplitude. The first condition can be met by defining two aperturegrating structures on the device facet with their respective orientation angles equal to $\theta= \pm 45^{\circ}$. Figure 3 is a schematic of the device. The distance between the aperture and the nearest groove on the left and right is denoted as $d_{1}$ and $d_{2}$, respectively. The grating period is tuned to be approximately equal to the SP wavelength $\lambda_{\mathrm{SP}}=9.4 \mu \mathrm{m}$ so that the reemissions from the grating grooves add up in phase in the direction normal to the facet. ${ }^{10}$ By tuning $d_{1}$ and $d_{2}$ such that $\left|d_{1}-d_{2}\right|$ approximately equals a quarter of $\lambda_{\mathrm{SP}}$, one can ensure that the two beams produced by the left and the right gratings as a result of the interference of the reemissions will have a $90^{\circ}$ phase difference. Simulation results shown in Fig. 4 can be used to determine the specific $d_{1}$ and $d_{2}$ so that the two beams have approximately the same amplitude. For ex-

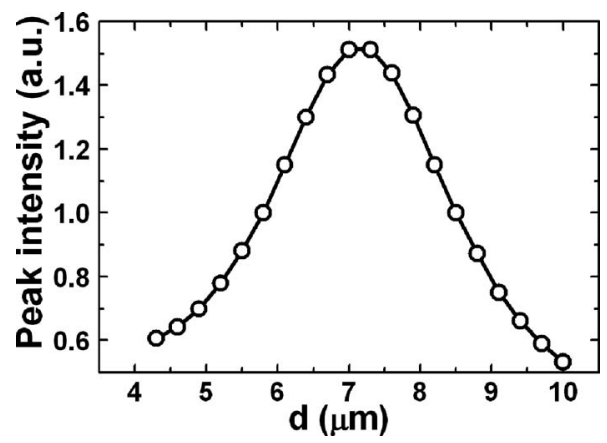

FIG. 4. Calculated peak intensity of the beam created by one grating vs the distance between the aperture and the first grating groove $d$. 


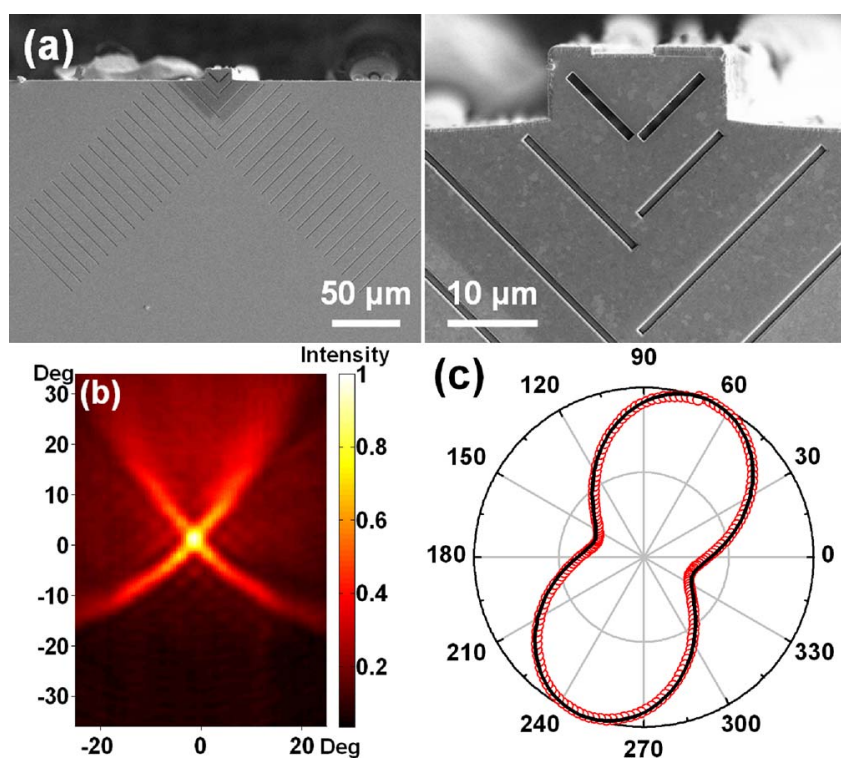

FIG. 5. (Color online) (a) Left panel: An electron micrograph of the facet of a QCL integrated with a plasmonic polarizer. There are two gratings orthogonal to each other, each containing 20 grooves. Right panel is the zoom-in view of the left panel. $d_{1}=8.3 \mu \mathrm{m}$ and $d_{2}=6.0 \mu \mathrm{m}$. (b) A measured 2D far-field emission pattern of the device. (c) Measured optical power of the central spot in (b) while a wire-grid polarizer was rotated in front of the detector (rotation angle $\alpha$ ). Open circles are experimental data and solid curve is a fitting according to $0.5 A^{2}+0.5 B^{2} \sin ^{2} \alpha$ $+A B \sin \alpha \sin \left(\alpha-\varphi+45^{\circ}\right)$. The first and second terms correspond to the circularly polarized and the linearly polarized components, respectively. The third term is due to interference of the two components.

ample, from Fig. 4, one finds that for a $\lambda=9.9 \mu \mathrm{m}$ QCL the two beams will have $90^{\circ}$ phase difference and the same amplitude if $d_{1}=6.0 \mu \mathrm{m}$ and $d_{2}=8.3 \mu \mathrm{m}$. The direct emissions from the two apertures have no phase difference, and they contribute to a TM polarized optical background.

Experimental results for a circularly polarized laser are presented in Fig. 5. Figure 5(a) shows electron micrographs of the device facet. A measured 2D far-field emission pattern of the device is shown in Fig. 5(b). Each grating contributes to a one-dimensionally collimated beam and the far-field pattern is cross shaped. Figure 5(c) is the measured device output when a wire-grid polarizer was rotated in front of a detector. The detector was placed in the far field corresponding to the center of the cross-shaped emission pattern. The analysis of Fig. 5(c) indicates that this part of the laser beam consists of a right circularly polarized component $A \cos (\omega t$ $+\varphi)(\hat{x}+\hat{z}) / \sqrt{2}+A \cos \left(\omega t+\varphi-90^{\circ}\right)(-\hat{x}+\hat{z}) / \sqrt{2}$ and a linearly polarized component $B \cos (\omega t) \hat{z}$, where $A$ and $B$ are electric field amplitude, $\hat{x}$ and $\hat{z}$ are unit vectors (see Fig. 1), and $\varphi$ and $\varphi-90^{\circ}$ are the phases accumulated while SPs propagate from the apertures to the first groove of the left and the right gratings, respectively. As discussed in the last paragraph, the circularly and linearly polarized components originate from the two gratings and the two apertures, respectively. We find that $A / B=1.4$ and $\varphi=350^{\circ}$ fit the experimental data with minimum errors, see Fig. 5(c). With the current design, the far field is a superposition of a circularly and a non- negligible linearly polarized components. We envision some improved designs, which offer efficient suppression of the linearly polarized component. For example, our simulations showed that in a design where the aperture is first covered by a piece of transparent dielectric and then a piece of metal, the direct output from the apertures can be entirely coupled into SPs.

A QCL with elliptically polarized light output can be constructed using a similar structure, as shown in Fig. 3. The amplitude and phase of the two beams originating from the two gratings are controllable by tuning $d_{1}$ and $d_{2}$. If their amplitude is different and/or if their phase difference is not $90^{\circ}$, the intersection of the two beams will be elliptically polarized. In a special case where the two beams have the same amplitude and a phase difference of $180^{\circ}$, the output light will be TE polarized with electric field in the plane of the waveguide layers ( $x$ direction in Fig. 1). In this case, the plasmonic polarizer functions as a half-wave plate. Integrated plasmonic polarizer offers a universal solution to the problem of manipulating the polarization state of a light beam. A similar design can be scaled to satisfy essentially all solid-state light sources with different wavelengths.

We acknowledge support from the Air Force Office of Scientific Research (AFOSR MURI on Plasmonics) and the Harvard Nanoscale Science and Engineering Center (NSEC). This work was performed in part at the Center for Nanoscale Systems (CNS) at Harvard University, a member of the National Nanotechnology Infrastructure Network (NNIN), which is supported by the National Science Foundation. N.Y. thanks Diandian Mao for creating the cover illustration based on this work for the current issue of Appl. Phys. Lett.

${ }^{1}$ W. L. Pritchard and J. A. Sciulli, Satellite Communication Systems Engineering (Prentice-Hall, Inc., New Jersey, 1986), Chap. 10, p. 333.

${ }^{2}$ N. Berova, K. Nakanishi, and R. W. Woody, Circular Dichroism: Principles and Applications, 2nd ed. (Wiley-VCH, New York, 2000), Chap. 1, p. 28.

${ }^{3}$ H.-K. Lo, S. Popescu, and T. Spiller, Introduction to Quantum Computation and Information (World Scientific, Singapore, 1998), p. 120.

${ }^{4}$ A. Yariv and P. Yeh, Photonics: Optical Electronics in Modern Communications, 6th ed. (Oxford University Press, Oxford, 2007), Chap. 16, p. 719.

${ }^{5}$ H. C. Liu and F. Capasso, Intersubband Transitions in Quantum Wells: Physics and Device Applications I (Academic, San Diego, 2000), Chap. 1, p. 8.

${ }^{6}$ R. Gordon, A. G. Brolo, A. McKinnon, A. Rajora, B. Leathem, and K. L. Kavanagh, Phys. Rev. Lett. 92, 037401 (2004).

${ }^{7}$ J. Elliott, I. I. Smolyaninov, N. I. Zheludev, and A. V. Zayats, Phys. Rev. B 70, 233403 (2004)

${ }^{8}$ A. Drezet, C. Genet, and T. W. Ebbesen, Phys. Rev. Lett. 101, 043902 (2008).

${ }^{9}$ H. J. Lezec, A. Degiron, E. Devaux, R. A. Linke, L. Martín-Moreno, F. J. García-Vidal, and T. W. Ebbesen, Science 297, 820 (2002).

${ }^{10}$ N. Yu, J. Fan, Q. J. Wang, C. Pflügl, L. Diehl, T. Edamura, M. Yamanishi, H. Kan, and F. Capasso, Nature Photon. 2, 564 (2008).

${ }^{11}$ X. Shi and L. Hesselink, J. Opt. Soc. Am. B 21, 1305 (2004).

${ }^{12}$ N. Yu, R. Blanchard, J. Fan, Q. J. Wang, C. Pflügl, L. Diehl, T. Edamura, M. Yamanishi, H. Kan, and F. Capasso, Opt. Express 16, 19447 (2008).

${ }^{13}$ N. Yu, R. Blanchard, J. Fan, T. Edamura, M. Yamanishi, H. Kan, and F. Capasso, Appl. Phys. Lett. 93, 181101 (2008). 